Z Epileptol $2011 \cdot 24: 5-5$

DOI 10.1007/s10309-010-0160-8

Online publiziert: 16. Januar 2011

(c) Springer-Verlag 2011

\author{
B. Steinhoff \\ Epilepsiezentrum Kork, Kehl-Kork
}

\title{
Epileptologische Labordiagnostik
}

Das erste Heft des Jahres 2011 steht wesentlich im Zeichen der epileptologischen Labordiagnostik. Anlass ist eine Originalarbeit in diesem Heft, die auf die laboranalytischen Probleme aufmerksam macht, die im Zusammenhang mit Sendungen biologischer Flüssigkeiten auftreten. Um diese Originalarbeit herum publizieren wir aktuelle Übersichten, die sich mit dem Gebiet der Serumkonzentrationen von Antikonvulsiva und der Labordiagnostik im weiteren Sinne beschäftigen. Das wichtige Thema entzündlicher Ursachen von Epilepsien ist ja erst vor Kurzem in dieser Zeitschrift kommentiert worden.

Dankbar bin ich auch dafür, dass - wie immer - rasch zumindest einige Kollegen zur Expertenfrage Stellung bezogen, die sich ebenfalls mit Blutspiegeln der Antikonvulsiva beschäftigt.
Ich hoffe sehr, dass Sie uns bei der Gestaltung der Zeitschrift für Epileptologie nicht im Stich lassen. Das wäre gerade jetzt ausgesprochen schade, da wir im Begriff sind, unserer epileptologischen Zeitschrift auch international Reputation zu verschaffen und im Jahr 2011 den Versuch zu unternehmen, eine Listung in Medline zu erreichen.

Drücken Sie uns die Daumen!

Bernhard J. Steinhoff (Kehl-Kork)

\section{Korrespondenzadresse}

Prof. Dr. B. Steinhoff

Epilepsiezentrum Kork

Landstr. 1, 77694 Kehl-Kork

bsteinhoff@epilepsiezentrum.de 